\title{
A educação física no âmbito do tratamento em saúde mental: um esforço coletivo e integrado*1
}

Lígia Gizely dos Santos Chaves Melo*2 Kleber Roberto da Silva Gonçalves de Oliveira*3 José Vasconcelos-Raposo*4

Este artigo analisa a Educação Física no processo de tratamento de doenças mentais, identificando as contribuições dessa prática profissional às terapias em saúde mental. Discute-se nesse estudo o papel da Educação Física enquanto atividade terapeutica auxiliar na diminuição da sintomatologia dessas doenças em função da aplicação de atividades físicas identificada em diversos estudos. Concluiu-se que a integração da Educação Física como ciência auxiliar à Psiquiatria tem se constituído um esforço interdisciplinar nos tratamentos de pacientes em sofrimento mental.

Palavras-chave: Educação física, saúde mental, interdisciplinaridade, tratamento

${ }^{* 1}$ Este texto constitui parte da dissertação sob o título Atividade física como complemento terapêutico multidisciplinar em saúde mental: estudo do impacto de um programa de exercício físico aeróbico nos sintomas negativos em esquizofrênicos hospitalizados, da Universidade de Trás-os-Montes e Alto Douro - UTAD, Vila Real, Portugal.

*2 Universidade de Trás-os-Montes e Alto Douro - UTAD (Vila Real, Portugal).

${ }^{*}$ Universidade Federal do Pará - UFPA (Belém, PA, Br)

${ }^{*}$ Universidade de Trás-os-Montes e Alto Douro - UTAD (Vila Real, Portugal). 


\section{Introdução}

A Educação Física é considerada uma área do conhecimento científico que, para fins de classificação dentro do saber acadêmico, em algumas universidades, encontra-se vinculada ao campo das Ciências Sociais e Humanas e, em outras, à área das Ciências da Saúde.

Historicamente, as raízes do conhecimento da Educação Física podem ser situadas no campo da Filosofia, Biologia e Sociologia. Atualmente, diante da inserção dos profissionais, principalmente a partir das duas últimas décadas, na saúde pública, potencializada pela política pública do Sistema Único de Saúde (SUS), do Movimento Antimanicomial (no campo da saúde mental) e no contexto das equipes dos Núcleos de Apoio de Saúde da Família (NASFs), a profissão tem sido caracterizada cada vez mais como pertencente à área da saúde.

Assim, o lugar da Educação Física em saúde mental possui parâmetros utilizados para situá-la em alguns contextos no campo das Ciências da Saúde. Esses parâmetros nos conduzem a dois questionamentos: A filiação da Educação Física à determinada área do conhecimento científico decorre dos espaços de intervenção profissional ou do objeto de estudo da profissão? A constituição da Educação Física como ciência já revela uma natureza interdisciplinar? Para responder a esses questionamentos optou-se por analisar pesquisas no Brasil e em escala internacional que pudessem vislumbrar a contribuição do objeto da Educação Física às práticas em saúde mental.

Este artigo tem por objetivo analisar o processo de integração da Educação Física no âmbito dos tratamentos em saúde mental de forma a identificar as contribuições dessa prática profissional às terapias relativas ao sofrimento mental. 


\section{SAÚDE MENTAL}

\section{As atividades físicas como fator da elevação do bem-estar do doente mental}

Para Berk (2007), o estilo de vida ocidental é predominantemente sedentário, ocasionando um grave problema de saúde que é uma premissa importante a ser observada no paciente esquizofrênico. Por outro lado Callaghan (2004) aponta que indivíduos com aptidão física melhorada pela prática de exercício físico apresentam muitos benefícios fisiológicos que os capacitam para execução de atividades cotidianas, sem dores, cansaço ou esgotamento físico, e Donaghy (2007) ressalta que, na perspectiva do sofrimento psíquico, a atividade física melhora proporcionalmente o nível de saúde mental.

O exercício físico está presente em diversas atividades como programas de treinamento desportivo, aulas de Educação Física, atividades de academia, sessões de reabilitação terapêutica, entre outros processos de intervenção motora. A efetividade desta gama de propostas em que o exercício físico encontra-se inserido é influenciada por fatores ambientais, materiais e qualidade técnica e científica dos profissionais envolvidos (Booth \& Roberts, 2008).

O exercício físico é recomendado para a população em geral por muitas entidades médicas, incluindo a American College of Sports Medicine (ACSM). É de notório conhecimento que a atividade física é considerada uma importante ferramenta para a melhoria da saúde pública e para o aprimoramento de qualidades físicas de acordo com o tipo de exercício executado (ACSM, 2010; WHO, 2006). Ela está diretamente relacionada à qualidade de vida em indivíduos saudáveis e relacionada à saúde para pessoas com diversas ordens de patologias como: artrite, câncer, cardiopatias, diabetes melito, incapacitações por paralisia cerebral e lesões na medula espinhal, dislipidemia, vírus da imunodeficiência humana, hipertensão, síndrome metabólica, sobrepeso e obesidade, osteoporose, doença arterial periférica e doença renal (ACSM, 2010).

Segundo Goodwin (2003) pouco mais da metade $(60,3 \%)$ de um total de 5.877 adultos nos Estados Unidos mantinham a prática regular de exercícios físicos e estes estavam relacionados à baixa prevalência de depressão e ansiedade. Este número é grandiosamente inferior na população acometida por transtorno mental (Dunn \& Jewell, 2010; Vasconcelos-Raposo, 2011). A população acometida por transtornos mentais morre 10-15 anos mais cedo do que a população em geral, e os principais fatores contribuintes incluem prevenção de doenças cardiovasculares resultantes de escolhas de estilo de vida pobre, como a inatividade física (Parks, Svendsen, Singer \& Foti, 2006).

Os transtornos mentais incluem sintomas emocionais e comportamentais definidas pelo Manual Diagnóstico e Estatístico de Transtornos Mentais - quarta 
edição (DSM-IV, 2000) e a Classificação Estatística Internacional de Doenças e Problemas Relacionados à Saúde - décima revisão (CID-10, 2008). Estes incluem esquizofrenia, depressão, transtornos de ansiedade, déficit de atenção e hiperatividade, transtorno de conduta e abuso e dependência de substâncias.

O exercício físico está inserido no Sistema Único de Saúde (SUS) com o objetivo de cumprir exigências das diretrizes na forma de ações e serviços de modo universal e integral da assistência com ações e serviços de prevenção e curativos, individuais e coletivos em todos os níveis de complexidade. No panorama da reforma psiquiátrica o exercício físico é incluído como uma terapia na recuperação em casos de transtornos psiquiátricos graves e manutenção para auxiliar no impedimento de novas crises e na prevenção para que o indivíduo não adoeça (Roeder, 2003).

$\mathrm{Na}$ prática acadêmica, os exercícios são propostos a pacientes com transtorno mental por várias razões (Amersfoort, 2004). Nesse sentido, há uma gama de pesquisas que relacionam a eficácia do exercício aos ganhos da aptidão física tanto de pacientes saudáveis quanto de pacientes psiquiátricos (Callaghan, 2004; Meyer \& Broocks, 2000). Os benefícios das alterações e adaptações bioquímicas e fisiológicas estão relacionados com a prática de exercícios regulares. Alguns destes mecanismos afetam o humor, por exemplo, por meio de serotonina (Wipfli, Landers, Nagoshi \& Ringenbach, 2011), as endorfinas que atuam como ansiolítico, ou alteram a reatividade ao estresse (hipotálamo-pituitária-adrenal) relacionado ao nível de cortisol e memória, dentre outros (Wolf, Schommer, Hellhammer, McEwen, \& Kirschbaum, 2001; Wolf, 2012).

Pesquisas revelam a eficácia da utilização do exercício enquanto terapia não medicamentosa coadjuvante para a saúde mental (Lincoln, Shepherd, Johnson, \& Castaneda-Sceppa, 2011; Have, 2011; Vasconcelos-Raposo, 2011) em pacientes com ansiedade (Berk, 2007; Goodwin, 2003; Wipfli et al., 2011), sintomas depressivos em pacientes com artrite (Dario, Külkamp, Faraco, Gevaerd \& Domenech, 2010), dependentes de álcool e drogas (Goodwin, 2003), depressão (Dunn, Trivedi, Kampert, Clark \& Chambliss, 2005; Helmich et al., 2010; Lincoln et al., 2011; Wipfli et al., 2011), distúrbios alimentares (Stathopoulou, Powers, Berry, Smits \& Otto, 2006) e esquizofrenia (Holley, Crone, Tyson \& Lovell, 2011).

De um modo abrangente, independente da patologia acometida no indivíduo, é observada a melhora dos sintomas de ansiedade (Goodwin, 2003; Meyer \& Broocks, 2000), autoconfiança (Meyer \& Broocks, 2000), autoestima (Durão, Souza \& Miasso, 2005; Sonstroem \& Morgan, 1989), depressão (Goodwin, 2003; Meyer \& Broocks, 2000), humor (Peluso \& Andrade, 2005), imagem corporal (Meyer \& Broocks, 2000) qualidade de vida relacionada à saúde (Schmitz, N., 2004). A melhora tanto em indivíduos saudáveis quanto em pacientes psiquiátricos torna $\mathrm{o}$ exercício uma ferramenta auxiliar na prevenção e tratamento de doenças psiquiátricas (Peluso \& Andrade, 2005). 


\section{SAÚDE MENTAL}

Porém, a prescrição inadequada de exercício físico pode desencadear problemas como a síndrome do overtraining (Petibois, Cazorla, Poortmans \& Deleris, 2002), dismofia muscular (Drewnowski, Kurth \& Krah, 1995), distúrbios no humor (Peluso \& Andrade, 2005), obsessão por exercício (Bamber, Cockerill, \& Carroll, 2000), alterações psiquiátricas causadas por anabolizantes (Peluso, Assunção, Araújo \& Andrade, 2000).

Os benefícios do exercício físico nas qualidades físicas dos pacientes esquizofrênicos são bem documentados na literatura e contribuem para o aumento da longevidade nesta população (Richardson et al., 2005). Pacientes com esquizofrenia apresentam níveis bastante inferiores de participação de atividade física regular quando comparados à população saudável por diversos fatores, dentre os quais o estigma, o desconhecimento da possibilidade de participação, fatores sociais, econômicos (Chamove, 1986; Daley, 2002; Doh, 2006).

É evidenciado que a atividade física também pode ser útil, nos mais graves problemas de saúde mental, como na esquizofrenia e psicose (Ellis, Crone, Davey \& Grogan, 2007; Faulkner, Cohn \& Remington. 2006). Com esta evidência, tem havido um aumento de pesquisas voltadas para a utilização da atividade física na saúde mental como um complemento ao tratamento e, em alguns casos, como parte integrante da abordagem plano de assistência à saúde (Crone, Heaney \& Owens, 2009).

Apesar da existência de pesquisas acerca dos efeitos terapêuticos do exercício na saúde mental (Vasconcelos-Raposo, 2011), deve-se levar em consideração a forma como tais pesquisas devem ser divulgadas aos profissionais de saúde mental (Callaghan, 2004; Faulkner \& Biddle, 2001) e aos gestores em saúde (Vasconcelos-Raposo, 2011), pois os profissionais de saúde, de um modo geral, somente recomendam o exercício físico para seus pacientes pelos aspectos positivos alcançados em relação à melhoria da aptidão física (Daley, 2002; Faulkner \& Biddle, 2001; Dunn \& Jewell, 2010), sendo negligenciada, pelos gestores, a importância do exercício físico enquanto ferramenta terapêutica (Vasconcelos-Raposo, 2011).

As pesquisas descritas revelam que a atividade física traz benefícios aos pacientes esquizofrênicos no sentido de propor uma dinâmica diferenciada a esses sujeitos, tornando-os agentes principais do tratamento, libertando-os da rotina dos remédios a que comumente são submetidos. A atividade física representa também uma possibilidade de alívio da ansiedade, pois a rotina de hospitais e unidades de saúde que cuidam de esquizofrênicos, por vezes impõe ao paciente uma rotina de terapias medicamentosas que falseiam o seu o quadro clínico.

As Diretrizes da Federação Mundial das Sociedades de Psiquiatria Biológica para o Tratamento Biológico da Esquizofrenia relatada por Falkai et al. (2006) na Revista de Psiquiatria Clínica mostram que a atividade física é muito importante para pacientes esquizofrênicos, uma vez que estes, em virtude da enfermidade, são mais propensos ao sedentarismo e com isso desenvolvem obesidade que traz 
risco de vida e que, segundo eles, tem se ampliado entre esses pacientes, tendo em vista que,

Indivíduos com esquizofrenia têm mais sobrepeso ou obesidade que os indivíduos da população geral (Marder et al., 2004). Combinado com outros fatores de risco (como tabagismo, atividade física reduzida, diabetes e dislipidemia), o risco de morbidade e mortalidade cardiovasculares se mostra aumentado. (Falkai et al., 2006, p. 22)

Essas referências mostram que realizar atividade física com pacientes esquizofrênicos implica também uma série de variáveis que devem ser identificadas pelo profissional destacado para aplicar essa tarefa. Gomes (2011) em dissertação de mestrado defendida na Faculdade de Desporto da Universidade do Porto sob o título "Atividade física e desportiva para indivíduos com esquizofrenia ou transtorno esquizoafetivo", a qual foi analisada a partir da implantação de um programa de capacidade funcional no qual a autora identificou que nem sempre a atividade física corrobora com a melhora do paciente, uma vez que as melhoras estão em função da inconstância do estado paciente. A autora relata que não identificou mudanças significativas na perda de peso ou da circunferência do abdome.

\section{A interferência da atividade física na sintomatologia do transtorno mental}

Algumas pesquisas sobre programas de exercícios físicos relacionados a sintomas negativos em pacientes esquizofrênicos mostram que os termos exercício físico e esquizofrenia, no Information Sciences Institute, foram encontradas 19 pesquisas relevantes entre o período de 1999 a 2011. Sendo que a maioria dos estudos relaciona os efeitos das qualidades físicas no público pesquisado. Enquanto que apenas três estudos relacionam o exercício físico com os sintomas da esquizofrenia.

Beebe et al. (2011) realizaram um estudo piloto com dez pacientes com alta hospitalar que participaram de um programa de caminhada de cinco a trinta minutos, três vezes por semana, durante o período de 16 semanas em que foram analisados a severidade dos sintomas psiquiátricos, índice de massa corporal e percentual de gordura. Não houve diferença significativa entre os sintomas negativos, positivos e escore total da Escala dos sintomas positivos e negativos (PANSS) entre grupo experimental e grupo controle.

No estudo de Marzolini, Jensen \& Melville (2009) 13 pacientes foram divididos em grupo experimental e controle onde o grupo experimental participou de um programa aeróbico e de resistência, sendo noventa minutos de duração por sessão, duas vezes por semana, durante 12 semanas, sendo avaliado o nível de 


\section{SAÚDE MENTAL}

saúde mental, a capacidade funcional de exercício, força muscular e medidas antropométricas. $\mathrm{O}$ grupo controle apresentou melhora significativa na pontuação total do inventário de saúde mental $(\mathrm{p}<0,03)$ quando comparado ao grupo controle.

Acil, Dogan \& Dogan (2008) realizaram uma pesquisa sobre o efeito de um programa de um exercício físico aeróbico, inicialmente por vinte e cinco minutos, duas vezes por semana à quarenta minutos por sessão, três vezes por semana, em um período de dez semanas, com uma amostra de trinta pacientes internados e de alta hospitalar, divididos, de modo igualitário, entre grupo experimental e controle com o objetivo de avaliar os sintomas positivos e negativos da esquizofrenia, utilizando como escalas avaliativas: o Inventário breve de sintomas, a escala de avaliação de sintomas positivos e escala de avaliação dos sintomas negativos e o "World Health Organization Quality of Life Scale - Turkish Version". A pesquisa demonstrou que exercício aeróbico moderado é um programa eficaz na diminuição dos sintomas psiquiátricos comparado ao grupo controle.

Tais estudos apresentaram uma lacuna do conhecimento que instigou a realização do estudo referente à tese de doutorado a qual este artigo é constituinte, ou seja, é evidente que os sintomas positivos são, na sua maioria, tratados pela medicação especificada, porém os sintomas negativos necessitam das terapias complementares e nenhum dos estudos ora apresentados especificam quais sintomas negativos apresentam melhora significativa ao aplicar um programa de exercício físico a esquizofrênicos.

É inquestionável o valor da atividade física no tratamento do paciente esquizofrênico. Apesar dos resultados mostrarem efeitos negativos dessas atividades no comportamento do paciente, a atividade física, como foi possível observar, causa efeito bastante positivo nos indivíduos, especialmente os benefícios relacionados à obesidade, superação do sedentarismo, mas tais resultados sempre dependem do estado em que se encontram os pacientes.

\section{Considerações finais}

As análises desses estudos mostram que a atividade física é uma alternativa viável para a melhoria da situação dos pacientes esquizofrênicos, revelando que a equipe que envolve o profissional da Educação Física tem garantido um bom êxito nas experiências que integram saberes profissionais específicos.

O caráter interdisciplinar dos tratamentos relacionados ao sofrimento mental tem colaborado com a consolidação da reforma psiquiátrica com a inserção de áreas, inicialmente a Psicologia que iniciou sua inserção na década de 1970 e atualmente a Educação Física tem ocupado lugares importantes apresentando 
alternativas viáveis a partir do seu objeto. Respondendo ao questionamento que se fez inicialmente, observou-se que a intervenção profissional da Educação Física tem propiciado a integração do seu objeto centrado nas manifestações do movimento humano com as necessidades dos pacientes esquizofrênicos, como pode ser observado nas investigações de Parks, Svendsen, Singer \& Foti (2006); Amersfoort (2004), Callaghan, (2004); Meyer \& Broocks (2000); Wipfli, Landers, Nagoshi \& Ringenbach (2011); Wolf, Schommer, Hellhammer, McEwen \& Kirschbaum (2001); Wolf (2012) e as demais pesquisas relatadas.

Sobre o questionamento da constituição da Educação Física como ciência revela sua natureza interdisciplinar, uma vez que as descrições revelam ampla capacidade, por meio das atividades propostas de determinarem diagnósticos eficazes, como foi possível verificar nos resultados de Berk 2007; Goodwin, 2003; Wipfli et al. (2011); Lincoln, Shepherd, Johnson \& Castaneda-Sceppa, (2011); Have, M. 2011; Vasconcelos-Raposo (2011); Dario, Külkamp, Faraco, Gevaerd \& Domenech (2010); Goodwin (2003); Dunn, Trivedi, Kampert, Clark \& Chambliss (2005); Helmich et al. (2010); Lincoln et al. (2011); Wipfli et al. (2011) e Stathopoulou, Powers, Berry, Smits \& Otto (2006), Holley, Crone, Tyson \& Lovell (2011) sobre depressão, distúrbios alimentares, dependência de drogas e álcool, ansiedade, esquizofrenia, entre outros sintomas.

A natureza interdisciplinar da Educação Física no âmbito do trabalho em saúde mental é um forte argumento que sustenta o objeto do presente estudo, uma vez que a Educação Física, Psicologia, Serviço Social, Terapia Ocupacional, Medicina, Enfermagem, com seus respectivos objetos de estudo - a natureza fenômeno psicopatológico e as múltiplas expressões —, encontram-se profundamente vinculadas, uma vez que as desigualdades e os processos de exclusão oriundos das condições de organização e reprodução da vida material são constituintes dos processos de produção de subjetividades assujeitadas e/ou livres as quais, por sua vez, constituem a lógica de (re)produção do próprio sistema, seja conformando-se e/ou resistindo a ele, na perspectiva de manutenção do instituído e/ ou da produção de movimentos e forças instituintes (Baremblitt, 1992).

A interdisciplinaridade é um campo de essencial importância para o presente estudo, tendo em vista que a prática do profissional de Educação Física na assistência em saúde mental no hospital geral permeia o domínio de conhecimentos que permitem comunicação entre os membros da equipe, contribuindo com sua especificidade para a resolutividade de problemas propostos. Segundo Almeida et al. (2010) destacam que a Reforma Psiquiátrica brasileira é produto de um processo social que se constrói cotidianamente, uma realidade concreta e presente, mas um modelo ainda em construção. Dessa forma, sendo a doença mental um processo biopsicossocial, exige a convergência de saberes disciplinares diversos que se integrem, o que se constitui um desafio para os profissionais que tratam da saúde mental, criar 


\section{SAÚDE MENTAL}

novas práticas nessa direção, fazendo-se necessária investigação e reflexão em relação aos tratamentos dos transtornos mentais.

\section{Referências}

Acil, A. A., Dogan, S., Dogan, O. (2008). The effects of physical exercises to mental state and quality of life in patients with schizophrenia. Journal of Psychiatric Mental Health Nursing, 15(10), 808-815.

Almeida, M. M., Schall, V. T., Martins, A. M., \& Modena, C. M. (2010). Representações dos cuidadores sobre a atenção na esquizofrenia. $P \operatorname{siC}, 41(1), 110-117$.

American College of Sports Medicine (ACMS) (2010). ACSM's Guidelines for Exercise Testing and Prescription. ( $8^{\mathrm{a}}$ ed.). Philadelphia: Lippincott Williams \& Wilkins.

Amersfoort, Y. V. (2004). Prescripción de ejercicio físico y salud mental. In J. R., Grima \& C. B. Calafat (Eds.), Prescripcion de ejercicio fisico para la salud, (pp. 303-340). Barcelona: Paidotribo.

Bamber, D., Cockerill, I. M. \& Carroll, D. (2000). The pathological status of exercise dependence. British Journal of Sports Medicine, 34(2), 125-132.

Baremblitt, G. F. (1992). Compêndio de análise institucional e outras correntes: teoria e prática (5a ed.). Belo Horizonte: Instituto Félix Guattari.

Baremblitt, G. F. (2009). Prefácio. In I. C. F. Passos, Reforma psiquiátrica: as experiências francesas e italianas. Rio de Janeiro: Fiocruz.

Beebe, L., Smith, K., Burk, R., McIntyre, K., Dessieux, O., Tavakoli, A., Velligan, D. (2011). Effect of a Motivational Intervention on Exercise Behavior in Persons with Schizophrenia Spectrum Disorders. Community Mental Health Journal, 47(6), 628-636.

Berk, M. (2007). Should we be targeting exercise as a routine mental health intervention? Acta Neuropsychiatrica, 19, 217-218.

Booth, F. W. \& Roberts, C. K. (2008). Linking performance and cronic disease risk: indices of physical performance are surrogates for health. British Journal of Sports Medicine, 42(12), 950-952.

Callaghan, P. (2004). Exercise: a neglected intervention in mental health care? Journal of Psychiatric and Mental Health Nursing, 11, 476-483.

Chamove, A. (1986). Positive short-term effects of activity on behaviour in chronic schizophrenic patients. British Journal of Clinical Psychology, 25(2), 125-133.

Crone, D., Heaney, L., \& Owens, C. (2009). Physical activity and mental health. In L. Dugdill, D. Crone, \& R. Murphy (Eds.), Physical activity and health promotion: evidenced-based approaches to practice, (pp. 198-212). London: Blackwell.

Daley, A. J. (2002). Exercise therapy and mental health in clinical populations: is exercise therapy a worthwhile intervention? Advances in Psychiatric Treatment, 8, 262-270.

Dario, A.B.; Külkamp, W., Faraco, H.C., Gevaerd, M.S. \& Domenech, S.C. (2010). Alterações psicológicas e exercício físico em pacientes com artrite reumatoide. Motricidade, 6(3), 21-30. 
Diagnostic and Statistical Manual of Mental Disorders (DSM-IV) (2000). (4 ${ }^{\mathrm{a}}$ ed.). text rev. Washington, DC: American Psychiatric Association.

DoH - Department of Health. (2006). Choosing health: Supporting the health needs of people with severe mental illness. London: Author.

Donaghy, M. E. (2000). Exercise can seriously improve your mental health: fact or fiction? Advances in Physiotherapy, 79, 76-88.

Drewnowski, A., Kurth, C.L. \& Krah, D. D. (1995). Effects of body image on dieting, exercise, and anabolic steroid use in adolescent males. International Journal of Eating Disorders, 17(4), 381-386.

Dunn, A. L., \& Jewell, J. S. (2010). The effect of exercise on mental health. Current Sports Medicine Reports, 9(4), 202-207.

Dunn, A. L., Trivedi, M. H., Kampert, J. B. Clark, C. G. \& Chambliss, H.O. (2005). Exercise treatment for depression: efficacy and dose response. The American Journal of Preventive Medicine, 28, 1-8.

Durão, A. M. S., Souza, M. C. B. \& Miasso, A. L. (2005). Grupo de acompanhamento de portadores de esquizofrenia. Revista Brasileira de Enfermagem, 58(5), 524-528.

Ellis, N., Crone, D., Davey, R. \& Grogan, S. (2007). Exercise interventions as an adjunct therapy for psychosis: a critical review. British Journal of Clinical Psychology, 46(1), 95-111.

Falkai, P., Wobrock, T., Lieberman, J., Glenthoj, B., Gattaz, W. F. \& Möller, H. J. (2006). Diretrizes da Federação Mundial das Sociedades de Psiquiatria Biológica para o Tratamento Biológico da Esquizofrenia. Revista de Psiquiatria Clínica, 33, 7-64.

Faulkner, G. \& Biddle, S. (2001). Exercise and mental health: It's just not psychology! Journal of Sports Sciences, 19(6), 433-444.

Faulkner, G., Cohn, T. \& Remington, G. (2006). Validation of a physical activity assessment tool for individuals with schizophrenia. Schizophrenia Research, 82(2-3), 225-231.

Gomes, E. A. (2011). Atividade física e desportiva para individuos com esquizofrenia ou transtorno esquizoafetivo: implantação de um programa e análise e aplicabilidade de um teste de capacidade funcional. Porto/PT: Faculdade de desporto da Universidade do Porto. 121p. (Dissertação de Mestrado).

Goodwin, R. D. (2003). Association between physical activity and mental disorders among adults in the United States. Preventive Medicine, 36, 698-703.

Have, M. de ten, Graaf, R. \& Monshouwer, K. (2011). Physical exercise in adults and mental health status Findings from the Netherlands Mental Health Survey and Incidence Study (NEMESIS). Journal of Psychosomatic Research, 71, 342-348.

Helmich, I., Latini, A., Sigwalt, A., Carta, M. G., Machado, S., Velasques, B., Budde, H. (2010). Draft for clinical practice and epidemiology in mental health neurobiological alterations induced by exercise and their impact on depressive disorders. Clinical Practice \& Epidemiology in Mental Health, 6, 115-125.

Holley, J., Crone, D., Tyson, P. \& Lovell, G. (2011). The effects of physical activity on psychological well-being for those with schizophrenia: a systematic review. British Journal of Clinical Psychology, 50, 84-105. 


\section{SAÚDE MENTAL}

Lincoln, A.K., Shepherd, A., Johnson, P.L., \& Castaneda-Sceppa, C. (2011). The impact of resistance exercise training on the mental health of older Puerto Rican adults with type 2 diabetes. The Journals of Gerontology, Series B: Psychological Sciences and Social Sciences, 66(5), 567-570.

Marder, S. R., Essok, S. M., Miller, A. L., Buchanan, R. W., Casey, D. E., Davis, J. M. ... Shon, S. (2004). Physical health monitoring of patients with schizophrenia. American Journal of Psychiatry, 161, 1334-1349.

Marzolini, S., Jensen, B., \& Melville, P. (2009). Feasibility and effects of a group-based resistance and aerobic exercise program for individuals with severe schizophrenia: a multidisciplinary approach. Mental Health and Physical Activity, 2(1), 29-36.

Meyer, T. \& Broocks, A. (2000). Therapeutic Impact of Exercise on Psychiatric Diseases: guidelines for Exercise Testing and Prescription. Sports Medicine, 30(4), 269-279.

Organização Mundial de Saúde (2008). Classificação estatística internacional de doenças e problemas relacionados à saúde (CID-10). $8^{\mathrm{a}}$ ed. 10 $10^{\mathrm{a}}$ revisão, São Paulo: Editora da Universidade de São Paulo.

Parks, J., Svendsen, D., Singer, P. \& Foti, M. E. (2006). Morbidity and mortality in people with serious mental illness. Alexandria (VA): National Association of State Mental Health Program Directors.

Peluso, M. A. M. \& Andrade, L. H. S. G. (2005). Physical activity and mental health: the association between exercise and mood. Clinics, 60(1), 61-70.

Peluso, M.A., Assunção, S., Araújo, L. A. \& Andrade, L. (2000). Alterações psiquiátricas associadas ao uso de anabolizantes. Revista de Psiquiatria Clínica, 27(4), 229-236.

Petibois, C., Cazorla, G., Poortmans, J.R. \& Deleris, G. (2002). Biochemical aspects of overtraining in endurance sports: a review. Sports Medicine, 32(13), 867-878.

Richardson, C. R., Faulkner, G., McDevitt, J., Skrinar, G. S., Hutchinson, D. S. \& Piette, J. D. (2005). Integrating physical activity into mental health services for persons with serious mental illness. Psychiatric Services, 56(3), 324-331.

Roeder, M. A. (2003). Atividade fisica, saúde mental \& qualidade de vida. Rio de Janeiro: Shape. p. 365.

Schmitz, N., Kruse, J. \& Kugler, J. (2004). The association between physical exercises and health-related quality of life in subjects with mental disorders: results from a cross-sectional survey. Preventive Medicine, 39, 1200-1207.

Sonstroem, R. J. \& Morgan, W. P. (1989). Exercise and self-esteem rationale and model. Medicine \& Science in Sports \& Exercise, 21, 329-337.

Stathopoulou, G., Powers, M. B., Berry, A. C., Smits, J. A. J., \& Otto, M. W. (2006). Exercise Interventions for Mental Health: a quantitative and qualitative review. Clinical Psychology: Science and Practice, 3(2), 179-193.

Vasconcelos-Raposo, J. (2011). Saúde e exercício físico como instrumento terapêutico: que papel para as revistas científicas? Motricidade, 7(2), 1-5.

Wipfli, B., Landers, D., Nagoshi, C. \& Ringenbach, S. (2011). An examination of serotonin and psychological variables in the relationship between exercise and mental health. Scandinavian Journal of Medicine \& Science in Sports, 21, 474-481. 
Wolf, O. T. (2012). Immediate recall influences the effects of pre-encoding stress on emotional episodic long-term memory consolidation in healthy young men. Stress, 15(3), 272-280.

Wolf, O. T., Schommer, N. C., Hellhammer, D. H., McEwen, B. S. \& Kirschbaum, C. (2001). The relationship between stress induced cortisol levels and memory differs between men and women. Psychoneuroendocrinology, 26, 711-720.

World Health Organization (WHO) (2006). The World Health Report 2006 - working together for health. Geneva: The World Health Report.

\section{Resumos}

(Physical education in mental health treatment: a collective and integrated project)

This article analyzes the use of physical education in treating mental illness by describing the contributions of this area to mental health therapies. Based on several studies, the authors discuss the benefits of physical education as a therapeutic aid in reducing the symptoms of psychic disorders. The conclusion is that physical education and science can be useful to psychiatry as part of interdisciplinary efforts in the treatment of patients with mental distress.

Key words: Physical education, mental health, interdisciplinary, treatment

(L'éducation physique et le traitement en santé mentale: un effort collectif et integré)

Cet article analyse le processus de l'éducation physique dans le traitement des maladies mentales en identifiant les apports de cette pratique professionnelle aux thérapies de la santé mentale. Nous discutons le rôle de l'éducation physique comme activité thérapeutique auxiliaire qui permet de réduire les symptômes de ces maladies à travers l'application d'activités physiques, identifié dans plusieurs études. Nous concluons que l'intégration de l'Éducation Physique comme support à la Psychiatrie représente un effort interdisciplinaire dans le traitement des patients souffrant de troubles mentaux.

Mots clés: Éducation physique, santé mentale, interdisciplinarité, traitement

(La educación física en el tratamiento de la salud mental: un esfuerzo colectivo e integrado)

En este artículo se analiza el proceso de la educación fisica en el tratamiento de las enfermedades mentales, identificando los aportes de esta práctica profesional en las terapias de salud mental. Se examina en este estudio el papel de la actividad de educación física como ayuda terapéutica en la reducción de los síntomas de estas enfermedades debido a la aplicación de las actividades fisicas identificadas en varios estudios. Se concluyó que la integración de la educación física como ciencia auxiliar 


\section{SAÚDE MENTAL}

a la psiquiatría ha constituido un esfuerzo interdisciplinario en el tratamiento de pacientes con dificultades mentales.

Palabras clave: Educación física, salud mental, interdisciplinaria, tratamiento

(Sportunterricht im Rahmen der Behandlung von Geistesstörungen: eine kollektive und integrierte Initiative)

Dieser Artikel untersucht den Sportunterricht im Behandlungsprozess von Geistesstörungen, wobei die positive Einwirkung dieser Berufspraxis auf die therapeutischen Behandlungen von Geisteskranken erforscht wird. Hier wird die Rolle sportlicher Aktivitäten als therapeutische Hilfsmittel diskutiert, die dazu beitragen, die Symptome dieser Krankheiten zu lindern, wie es in verschiedenen Studien festgestellt wurde. Daraus wird geschlossen, dass die Integration von Sportunterricht als ergänzende Wissenschaft in die Psychiatrie sich als wirksames interdisziplinäres Medium zur Behandlung von geistesgestörten Patienten erwiesen hat.

Schlüsselwörter: Sportunterricht im Rahmen

Citação/Citation: Melo, L. G. do S. C., Oliveira, K. R. da S. G. \& Vasconcelos-Raposo, J. (2014, setembro). A educação física no âmbito do tratamento em saúde mental: um esforço coletivo e integrado. Revista Latinoamericana de Psicopatologia Fundamental, 17(3), 501-514.

Editor do artigo/Editor: Profa. Dra. Ana Cristina Figueiredo

Recebido/Received: 12.11.2013/ 11.12.2013 Aceito/Accepted: 25.1.2014 / 1.25.2014

Copyright: (C) 2009 Associação Universitária de Pesquisa em Psicopatologia Fundamental/ University Association for Research in Fundamental Psychopathology. Este é um artigo de livre acesso, que permite uso irrestrito, distribuição e reprodução em qualquer meio, desde que o autor e a fonte sejam citados / This is an open-access article, which permits unrestricted use, distribution, and reproduction in any medium, provided the original author and source are credited. 
Financiamento/Funding: Os autores declaram não ter sido financiados ou apoiados / The authors have no support or funding to report.

Conflito de interesses/Conflict of interest: Os autores declaram que não há conflito de interesses / The authors declare that has no conflict of interest.

\section{Lígia Gizely dos Santos Chaves Melo}

Professora de Educação Física; Acadêmica do doutoramento em Ciências do Desporto da Universidade de Trás-os-Montes e Alto Douro (UTAD), Vila Real, Portugal.

Rodovia Br 316, km 2, 1865

67013-770 Ananindeua, PA, Br

e-mail: ligia_chaves@hotmail.com

\section{Kleber Roberto da Silva Gonçalves de Oliveira}

Médico; Psiquiatra; Professor Auxiliar II na área de Habilidades Médicas da Universidade Federal do Pará - UFPA (Belém, PA, Br).

Av. Marquês de Herval, 2381/303

66000-000 Belém, PA, Br

e-mail: drkrsgo@ig.com.br

\section{JosÉ VASCONCELOS-RAPOSO}

Doutor em Psicologia do Desporto; Professor Catedrático em psicologia do desporto, exercício e saúde da Universidade de Trás-os-Montes e Alto Douro (UTAD), Vila Real, Portugal.

Rua Dr. Manuel Carmona

5000-558 Vila Real, Portugal

e-mail: j.vasconcelos.raposo@gmail.com 